\title{
Exponentially tapered Josephson flux-flow oscillator
}

Benabdallah, A.; Caputo, J. G.; Scott, Alwyn C.

Published in:

Physical Review B

Link to article, DOI:

10.1103/PhysRevB.54.16139

Publication date:

1996

Document Version

Publisher's PDF, also known as Version of record

Link back to DTU Orbit

Citation (APA):

Benabdallah, A., Caputo, J. G., \& Scott, A. C. (1996). Exponentially tapered Josephson flux-flow oscillator. Physical Review B, 54(22), 16139-16146. https://doi.org/10.1103/PhysRevB.54.16139

\section{General rights}

Copyright and moral rights for the publications made accessible in the public portal are retained by the authors and/or other copyright owners and it is a condition of accessing publications that users recognise and abide by the legal requirements associated with these rights.

- Users may download and print one copy of any publication from the public portal for the purpose of private study or research.

- You may not further distribute the material or use it for any profit-making activity or commercial gain

- You may freely distribute the URL identifying the publication in the public portal

If you believe that this document breaches copyright please contact us providing details, and we will remove access to the work immediately and investigate your claim. 


\title{
Exponentially tapered Josephson flux-flow oscillator
}

\author{
A. Benabdallah and J. G. Caputo \\ Laboratoire de Mathématiques, Institut National de Sciences Appliquées \\ and Unité de Recherche Associee au Centre National de la Recherche Scientifique 1378, \\ Boite Postale 8, 76131 Mont-Saint-Aignan cedex, France
}

A. C. Scott

Department of Mathematics, University of Arizona, Tucson, Arizona, 85721 and Institute of Mathematical Modelling, Technical University of Denmark, Lyngby, Denmark

(Received 27 March 1996; revised manuscript received 26 June 1996)

\begin{abstract}
We introduce an exponentially tapered Josephson flux-flow oscillator that is tuned by applying a bias current to the larger end of the junction. Numerical and analytical studies show that above a threshold level of bias current the static solution becomes unstable and gives rise to a train of fluxons moving toward the unbiased smaller end, as in the standard flux-flow oscillator. An exponentially shaped junction provides several advantages over a rectangular junction including: (i) smaller linewidth, (ii) increased output power, (iii) no trapped flux because of the type of current injection, and (iv) better impedance matching to a load. [S0163-1829(96)00646-7]
\end{abstract}

\section{INTRODUCTION}

The unidirectional flow of magnetic flux quanta in long Josephson junctions provides a means for tunable oscillators at frequencies above $100 \mathrm{GHz}$ with power outputs of a few microwatts, ${ }^{1}$ but the spectral linewidths $(\Delta f / f)$ of about $10^{-5}$ are unacceptably large. ${ }^{2}$ This spectral width can be due to junction inhomogeneities, perturbations caused by trapped flux, and chaos in the dynamic behavior of the fluxon chain. ${ }^{3}$ Currently available possibilities for reducing the linewidth are to mix two such devices or to drive one by an external oscillator, ${ }^{3}$ but neither approach is entirely satisfactory. In this work we present a way of operating the flux-flow oscillator that eliminates some of the possible causes of large spectral width.

The device we propose shown in Fig. 1 consists of a junction with an exponentially tapered width, decreasing toward the load. The junction is preceded by a region where the oxide layer is thicker preventing the tunneling of Cooper pairs, usually called the "idle region.', When a bias current is applied to one end of the junction, flux moves toward the opposite end of the junction. The idle region damps out the fluctuations of this bias current, acting as a "fluxon reservoir' and also ensures that the edge of the junction is well defined and protected from atmospheric degradation so that it yields a uniform current density. We also avoid the problem of trapped flux because when the current is injected only at one end, the only static solution has a half fluxon content. Because of the absence of an external magnetic field as opposed to the standard flux-flow design, we can eliminate the perturbations due to the current inducing the magnetic field. Numerical studies show that a chaotic regime that exists for a rectangular junction can be avoided by choosing an exponential shape. This design also provides better impedance matching to an output load. ${ }^{4}$ After introducing the model in Sec. II, the static and dynamic solutions are discussed in Sec. III using energy arguments. Section IV presents a variety of numerical results, comparing exponential and rectangular geometries.

The use of exponential tapering in nonlinear wave dynamics was suggested in the late 1960's by Lindgren and Buratti in connection with the neuristor, an electronic model for nerve. ${ }^{5}$ For static structures, as Thompson's classic book reminds us, the principle of exponential tapering has long been employed, one of the most noted examples being Gustave Eiffel's famous tower. ${ }^{6}$

\section{MODEL FOR THE EXPONENTIALLY SHAPED JOSEPHSON JUNCTION}

The evolution of the phase inside the junction is given by the two-dimensional sine-Gordon equation ${ }^{8}$

$$
\left(1+\beta \frac{\partial}{\partial t}\right) \Delta \phi-\frac{\partial^{2} \phi}{\partial t^{2}}-\alpha \frac{\partial \phi}{\partial t}=\sin \phi,
$$

where the lengths have been normalized to the Josephson characteristic length, time has been normalized by the plasma frequency, $\alpha$ is the damping due to the normal electrons, and $\beta$ is the surface damping. ${ }^{7}$ The boundary condition connecting the normalized external magnetic field (or current) $\eta$ to the phase gradient is ${ }^{4}$

$$
\eta=-\left(1+\beta \frac{\partial}{\partial t}\right) \nabla \phi \times \hat{z} .
$$

Following Pagano et l. $^{4}$ we assume that the edge of the junction is given by two functions of the longitudinal dimension $x, f_{1}(x)$, and $f_{2}(x)$ so that the width of the junction is $w(x)=f_{1}(x)-f_{2}(x)$ as shown in Fig. 1. Equation (2) can be projected on the tangents to the curves $y=f_{1}(x), y=f_{2}(x)$ to yield

$$
\begin{aligned}
& \eta_{x}+f_{1}^{\prime} \eta_{y}=-\left(1+\beta \frac{\partial}{\partial t}\right)\left(\frac{\partial \phi}{\partial y}-f_{1}^{\prime} \frac{\partial \phi}{\partial x}\right), \\
& \eta_{x}+f_{2}^{\prime} \eta_{y}=-\left(1+\beta \frac{\partial}{\partial t}\right)\left(\frac{\partial \phi}{\partial y}-f_{2}^{\prime} \frac{\partial \phi}{\partial x}\right) .
\end{aligned}
$$




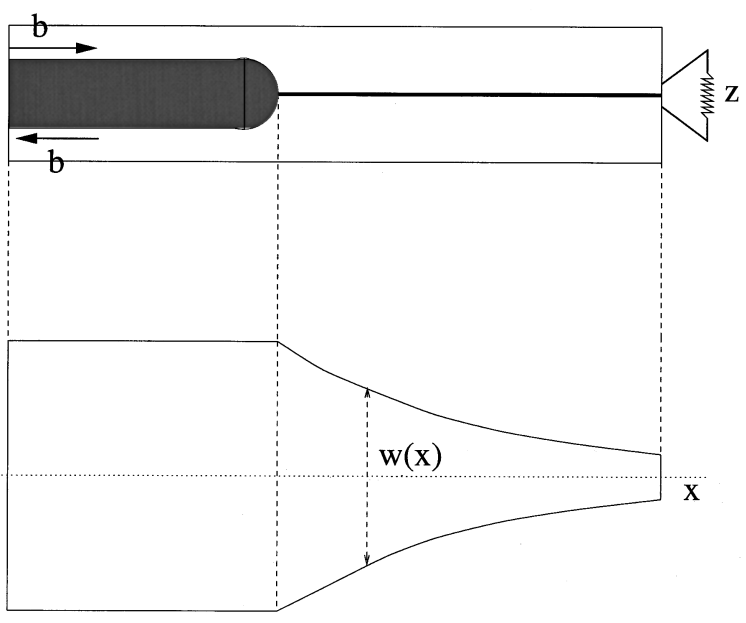

FIG. 1. An exponentially shaped junction together with its idle region and the external load $z$.

Now assume that the junction is narrow compared with $\lambda_{j}$, so that $w(x) \ll 1$ and the $y$ dependence of the phase can be neglected. Introducing the average along $y$

$$
\widetilde{\phi}(x)=\frac{1}{w(x)} \int_{f_{1}(x)}^{f_{2}(x)} \phi(x, y) d y,
$$

and assuming that $w(x)$ varies on scales larger than unity, the sine-Gordon equation (1) becomes

$$
\begin{aligned}
(1+ & \left.\beta \frac{\partial}{\partial t}\right)\left[\frac{\partial^{2} \widetilde{\phi}}{\partial x^{2}}+\frac{1}{w}\left(\left.\frac{\partial \phi}{\partial y}\right|_{y=f_{1}}-\left.\frac{\partial \phi}{\partial y}\right|_{y=f_{2}}\right)\right]-\frac{\partial^{2} \widetilde{\phi}}{\partial t^{2}} \\
& =\alpha \frac{\partial \widetilde{\phi}}{\partial t}+\sin \widetilde{\phi}
\end{aligned}
$$

Subtracting the projections of the boundary relations (3), (4), we get

$$
\begin{aligned}
\left(1+\beta \frac{\partial}{\partial t}\right)\left(\left.\frac{\partial \phi}{\partial y}\right|_{y=f_{1}}-\left.\frac{\partial \phi}{\partial y}\right|_{y=f_{2}}\right) \\
=\left.\eta_{x}\right|_{y=f_{2}}-\left.\eta_{x}\right|_{y=f_{1}}+\left.f_{2}^{\prime}(x) \eta_{y}\right|_{y=f_{2}}-\left.f_{1}^{\prime}(x) \eta_{y}\right|_{y=f_{1}} \\
+\left(1+\beta \frac{\partial}{\partial t}\right)\left[\left.f_{1}^{\prime}(x) \frac{\partial \phi}{\partial x}\right|_{y=f_{1}}-\left.f_{2}^{\prime}(x) \frac{\partial \phi}{\partial x}\right|_{y=f_{2}}\right] .
\end{aligned}
$$

Introducing the external current

$$
\gamma(x)=\frac{\left.\eta_{x}\right|_{y=f_{2}}-\left.\eta_{x}\right|_{y=f_{1}}}{w(x)}
$$

and the order 1 approximations

$$
\begin{gathered}
\left.f_{2}^{\prime}(x) \eta_{y}\right|_{y=f_{2}}-\left.f_{1}^{\prime}(x) \eta_{y}\right|_{y=f_{1}}=-\eta_{y}(x, 0, t) w^{\prime}(x), \\
\left(1+\beta \frac{\partial}{\partial t}\right)\left[\left.f_{1}^{\prime}(x) \frac{\partial \phi}{\partial x}\right|_{y=f_{1}}-\left.f_{2}^{\prime}(x) \frac{\partial \phi}{\partial x}\right|_{y=f_{2}}\right] \\
=\left(1+\beta \frac{\partial}{\partial t}\right) \frac{\partial \phi}{\partial x}(x, 0, t) w^{\prime}(x),
\end{gathered}
$$

we obtain the modified sine-Gordon equation ${ }^{4}$

$$
\begin{aligned}
(1 & \left.+\beta \frac{\partial}{\partial t}\right) \frac{\partial^{2} \widetilde{\phi}}{\partial x^{2}}+\frac{w^{\prime}(x)}{w(x)}\left(1+\beta \frac{\partial}{\partial t}\right) \frac{\partial \widetilde{\phi}}{\partial x}-\eta_{y} \frac{w^{\prime}(x)}{w(x)}+\gamma(x) \\
& =\sin \widetilde{\phi}+\frac{\partial^{2} \widetilde{\phi}}{\partial t^{2}}+\alpha \frac{\partial \widetilde{\phi}}{\partial t}
\end{aligned}
$$

In the following discussion, we shall assume an exponentially shaped junction as shown in Fig. 1, where

$$
f_{1}(x)=-f_{2}(x)=w_{0} \exp (-\lambda x)
$$

and

$$
w(x)=2 w_{0} \exp (-\lambda x)
$$

We also assume that there is no bias current so $\gamma(x)=0$ and $\eta_{y}=0$. Then the final equation obtained from Eq. (10) takes the simple form

$$
\frac{\partial^{2} \phi}{\partial t^{2}}-\frac{\partial^{2} \phi}{\partial x^{2}}+\sin \phi=-\alpha \frac{\partial \phi}{\partial t}-\lambda \frac{\partial \phi}{\partial x},
$$

where the tildes have been omitted and $\beta=0$.

Compared with the standard sine-Gordon model for Josephson junctions, this equation has the extra term $-\lambda \partial \phi / \partial x$, which corresponds to a force driving the fluxons toward the right. This is consistent with the fact that no static multifluxon solutions exist for decreasing width junctions, because as soon as a fluxon is created it tends to move in the direction of decreasing junction width in order to minimize its field energy. ${ }^{12}$

The bias current $b$ is applied on the left-hand end of the junction and we assume that the right-hand end is connected to a passive load of impedance $z$, which will absorb the fluxon chain. Then the boundary conditions at $x=0$ and $x=l$ are

$$
\begin{gathered}
\frac{\partial \phi}{\partial x}(0, t)=b, \\
-\frac{\partial \phi / \partial t}{\partial \phi / \partial x}(l, t)=z .
\end{gathered}
$$

\section{THE CONDITION OF MATCHED LOAD IMPEDANCE}

Assuming a traveling-wave solution $\phi(x, t)=f(x-u t)$ for the modified sine-Gordon equation (11) implies

$$
f^{\prime \prime}\left(1-u^{2}\right)-(\lambda-\alpha u) f^{\prime}=\sin f,
$$

which shows that any traveling-wave solution of the pure sine-Gordon equation is also a solution of Eq. (11) with the velocity

$$
u=\frac{\lambda}{\alpha} .
$$

The condition for impedance matching is obtained by equating this limiting velocity to

$$
-\left.\frac{\partial \phi / \partial t}{\partial \phi / \partial x}\right|_{x=l}=z
$$

where the optimum load $z=\lambda / \alpha$, a real number. Thus for an exponentially shaped junction the impedance can be exactly matched, which is not the case for a rectangular junction. We 
shall see that this fact has important consequences for the dynamic behavior of a flux-flow oscillator.

It is possible to obtain some information on the behavior of the velocity as it approaches the steady speed $\lambda / \alpha$ by examining the variation of the energy of the sine-Gordon equation due to the perturbation. To this end we multiply Eq. (11) by $\partial \phi / \partial t$ and integrate between 0 and $l$ to obtain

$$
\begin{gathered}
\frac{\partial}{\partial t} \int_{0}^{l}\left[\frac{1}{2}\left(\frac{\partial \phi}{\partial t}\right)^{2}+(1-\cos \phi)\right] d x-\int_{0}^{l} \frac{\partial^{2} \phi}{\partial x^{2}} \frac{\partial \phi}{\partial t} d x \\
=-\alpha \int_{0}^{l}\left(\frac{\partial \phi}{\partial t}\right)^{2} d x-\lambda \int_{0}^{l} \frac{\partial \phi}{\partial x} \frac{\partial \phi}{\partial t} d x,
\end{gathered}
$$

Integrating by parts the second term in the left-hand side

$$
\begin{aligned}
\int_{0}^{l} \frac{\partial^{2} \phi}{\partial x^{2}} \frac{\partial \phi}{\partial t} d x & =\left[\frac{\partial \phi}{\partial x} \frac{\partial \phi}{\partial t}\right]_{0}^{l}-\int_{0}^{l} \frac{\partial \phi}{\partial x} \frac{\partial^{2} \phi}{\partial x \partial t} d x \\
& =\left[\frac{\partial \phi}{\partial x} \frac{\partial \phi}{\partial t}\right]_{0}^{l}-\frac{1}{2} \frac{\partial}{\partial t} \int_{0}^{l}\left(\frac{\partial \phi}{\partial x}\right)^{2} d x,
\end{aligned}
$$

recalling the sine-Gordon Hamiltonian

$$
H=\int_{0}^{l}\left[\frac{1}{2}\left(\frac{\partial \phi^{2}}{\partial t}+\frac{\partial \phi^{2}}{\partial x}\right)+(1-\cos \phi)\right] d x,
$$

and using the boundary conditions (12) and (13), we obtain

$$
\begin{aligned}
\frac{d H}{d t}= & -b \frac{\partial \phi}{\partial t}(0, t)-z\left(\frac{\partial \phi}{\partial x}\right)^{2}(l, t)-\alpha \int_{0}^{l}\left(\frac{\partial \phi}{\partial t}\right)^{2} d x \\
& -\lambda \int_{0}^{l} \frac{\partial \phi}{\partial x} \frac{\partial \phi}{\partial t} d x .
\end{aligned}
$$

Consider each term on the right-hand side of Eq. (16). The first term gives the input power from the bias current, and the second term gives the ouput power into the load. The third term which is negative, indicates the power lost from internal damping. Finally consider the last term. Since $\phi_{t}=-u \phi_{x}$ for a wave that travels in the $x$ direction (toward the smaller end), this term is $\lambda \int_{0}^{l} u \phi_{x}^{2} d x$, which shows how the decreasing width of the junction in the $x$ direction increases the output power.

Assuming a single fluxon solution for which

$$
\phi(x, t)=4 \arctan \left[\exp \left(\frac{x-u t}{\sqrt{1-u^{2}}}\right)\right]
$$

and

$$
\frac{\partial \phi}{\partial x}=\frac{2}{\sqrt{1-u^{2}}} \operatorname{sech}\left(\frac{x-u t}{\sqrt{1-u^{2}}}\right),
$$

we substitute into Eq. (16) to obtain the modulation of the velocity by the perturbation as ${ }^{9}$

$$
\dot{u}=\left(1-u^{2}\right)(\lambda-\alpha u) .
$$

This equation shows that for $\lambda>\alpha$ the fluxon will always be accelerated toward the limiting value $u=1$. When $\lambda<\alpha$, the fixed point $u=\lambda / \alpha$ is linearly stable. When examining the linearization of Eq. (18) around the fixed point we obtain a typical convergence time $t_{\text {conv }}=\alpha /\left(\alpha^{2}-\lambda^{2}\right)$.
From this analysis, we expect that an exponentially shaped junction will have a different dynamic behavior from that of a rectangular junction. To examine this behavior in detail, we have undertaken a systematic numerical study of the modified sine-Gordon equation (11).

\section{NUMERICAL RESULTS}

To numerically integrate Eq. (11), we have transformed it into the system of coupled first-order equations

$$
\phi_{t}=\psi, \quad \psi_{t}=\phi_{x x}-\sin (\phi)-\alpha \psi-\lambda \phi_{x},
$$

which has been solved using a method of lines, where the time operator is advanced using an ordinary differential equation solver and the right-hand side is discretized using finite differences. We have chosen a junction of length $l=10$ and used 300 discretization points unless explicitly stated. The system is started with $\phi=0$ and $\partial \phi / \partial t=0$ and zero bias current. The bias current $b$ is then increased adiabatically so, allowing the solution to stabilize. We have chosen the damping parameter $\alpha=0.05$ which is the value typically used for flux-flow devices. ${ }^{4}$ Throughout the numerical experiments we will compare the behavior of a rectangular junction $(\lambda=0)$ with the one for an exponentially shaped junction with $\lambda=0.02$, this value was chosen to have an adiabatic tapering of the width. We have also examined the influence of the property of impedance matching Eq. (14) on the average output power and we show that in all cases the exponentially shaped junction is more advantageous.

For small $b$, no voltage is present in the system, corresponding to a time-independent solution for $\phi$. In this case, the time derivatives in the sine-Gordon equation (11) disappear so we obtain the modified pendulum equation

$$
\frac{d^{2} \phi}{d x^{2}}=\sin \phi+\lambda \frac{d \phi}{d x}
$$

together with the boundary conditions

$$
\left.\frac{d \phi}{d x}\right|_{x=0}=b
$$

and

$$
\left.\frac{d \phi}{d x}\right|_{x=l}=0 .
$$

If $\lambda=0$, this equation can be integrated as shown in the Appendix to yield the solution

$$
\Phi(x)=-2 \arctan \left(\frac{1}{\sinh \left(x-x_{\pi}\right)}\right),
$$

where

$$
x_{\pi}=\ln \left(\frac{2}{b}-\sqrt{\frac{4}{b^{2}}-1}\right) .
$$

The boundary condition Eq. (22) implies that there is only one type of solution which from the definition of $x_{\pi}$ is shown to disappear $b>b_{m}=2$. This solution has a fluxon content of one half and gives rise to a full fluxon because of the instability of the position $\phi=0$ as seen in the $\left(\phi, \phi_{x}\right)$ phase space in Fig. 14. Figure 2 shows the solution obtained numerically 


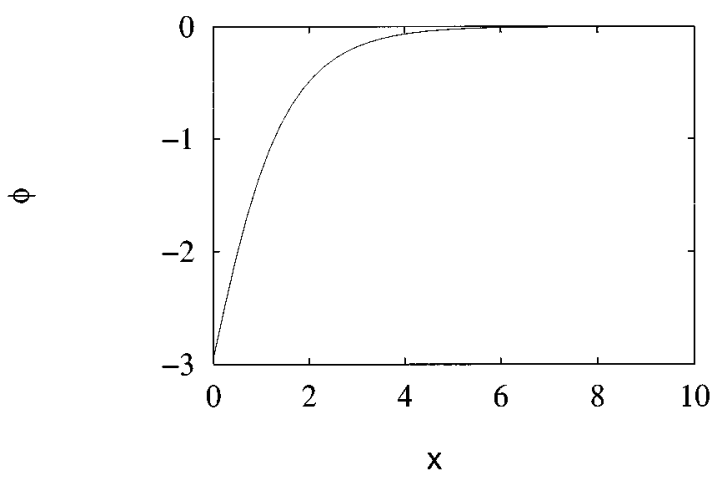

FIG. 2. The static solution for a rectangular junction $b=1.99$ (full line), the parameters are $\lambda=0, \alpha=0.05, z=0.2, l=10$, the number of discretization points is $n=300$. The analytic expression (23) is given in dashed lines.

and from Eq. (23) for $b=1.99$ in dashed line which completely overlaps the numerical result.

In the case $0<\lambda \ll 1$, a simple investigation in the $(\widetilde{\psi}, \phi)$ phase plane (where $\widetilde{\psi} \equiv d \phi / d x)$ given in the Appendix shows that the maximum value of $b$ for a static solution to exist is

$$
b_{m}=2-2 \lambda+\mathrm{O}\left(\lambda^{2}\right),
$$

which is confirmed by the plot in Fig. 3. Physically, this decrease in the threshold bias current for flux motion is caused by the force $\lambda d \phi / d x$, which tends to pull the fluxons toward the narrow end of the junction as soon as they are created.

When the bias current $b$ is increased past the threshold value, the static solution becomes unstable and gives rise to a dynamic state corresponding to a nonzero voltage. The phase observed in the middle of the junction for such a timedependent behavior (with $b=3.1$ ) is presented in the top of Fig. 4 for a rectangular junction and in the bottom of Fig. 4 for the exponentially shaped junction $(\lambda=0.02)$. Notice the well defined slope corresponding to a nonzero average voltage and the ripples in the rectangular case indicating an irregular behavior. By varying the bias current $b$ one can generate a continuum of different values of the voltage-and frequency-making this device a tunable oscillator.

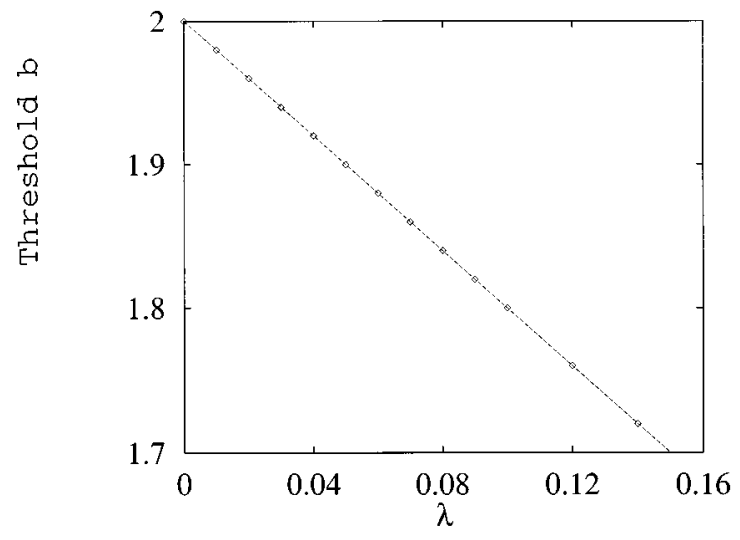

FIG. 3. Threshold value for $b$ as a function of the exponent $\lambda$. The parameters are the same as in Fig. 2.

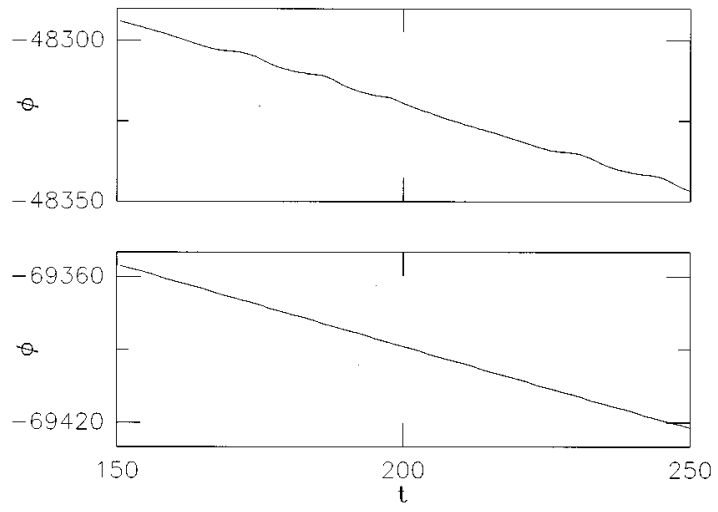

FIG. 4. Phase in the middle of the junction vs time. The bias current is $b=3.1$, the other parameters are the same as in Fig. 2. The top picture corresponds to the rectangular junction $(\lambda=0)$, while the bottom one is for an exponentially shaped junction $(\lambda=0.02)$.

Figure 5 shows such a $b-v$ characteristic for the rectangular junction (full line) and the exponentially shaped junction (dashed line). It can be seen that the threshold value for $b$ is lower for the exponential junction and also that the curve has smaller slope so that a small change in bias current will lead to a smaller change in voltage and frequency than for the rectangular junction, making the exponential junction oscillator easier to tune. The small dashed curve plotted close to the voltage axis is the first zero-field step in the $i-v$ characteristic obtained in the overlap geometry for this junction. We have plotted it to show that the voltage range spanned by the device involves the high-frequency domain of interest for applications.

The irregular behavior observed for the rectangular junction can be analyzed by examining the solution for different times. Figures 6 and 7 show the local velocity defined by

$$
-\frac{\partial \phi / \partial t}{\partial \phi / \partial x}
$$

for the rectangle and the exponential, which show randomness for the former and spatial coherence for the latter. Therefore it seems that the exponential shape contributes to

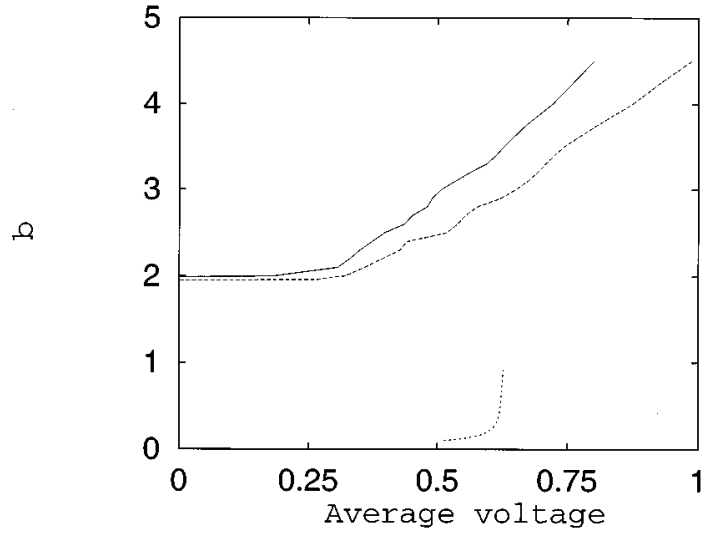

FIG. 5. Characteristic $b$ vs average voltage for the rectangular junction (full line), an exponentially shaped junction $\lambda=0.02$ (dashed line). The standard $i-v$ characteristic obtained for the rectangle in the overlap geometry is given for reference (light dash). 


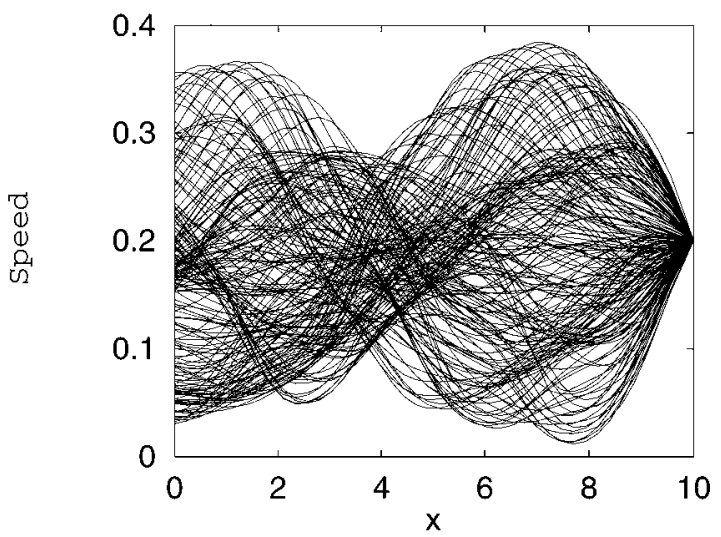

FIG. 6. Rectangular junction: local speed $(\partial \phi / \partial t) /(\partial \phi / \partial x)$ as a function of $x$ for different times, same parameters as in Fig. 4.

selecting a smaller number of spatial modes for the solution. The fact that the solution is chaotic for the rectangle has been mentioned as a possible cause for large spectral width, ${ }^{2}$ and we see that this effect is eliminated by using the exponential profile. That the solution is indeed chaotic for the rectangle can be better seen in Fig. 8 which shows the voltage in the middle of the junction as a function of time. For the exponential profile, on the other hand, the voltage is periodic as shown in Fig. 9.

Figures 10 and 11 show the associated power spectra, and there is clearly a large amount of low-frequency broadband noise for the rectangle, a signature of chaos. This dynamic behavior has been checked by doubling the number of spatial grid points, and no noticeable changes have been observed. The irregularity of the solution has the important consequence of a smaller average output power in the load as can be seen in Fig. 12, which shows $z(\partial \phi / \partial x)^{2}(l, t)$. Notice the large excursions observed for the rectangular junction as opposed to the periodic behavior for the exponential profile.

It is important for practical applications to know the influence of the load $z$ on the average output power of the device, in particular to see how it behaves for large loads which is the experimental situation. Figure 13 shows the dependency of the average output power as a function of the

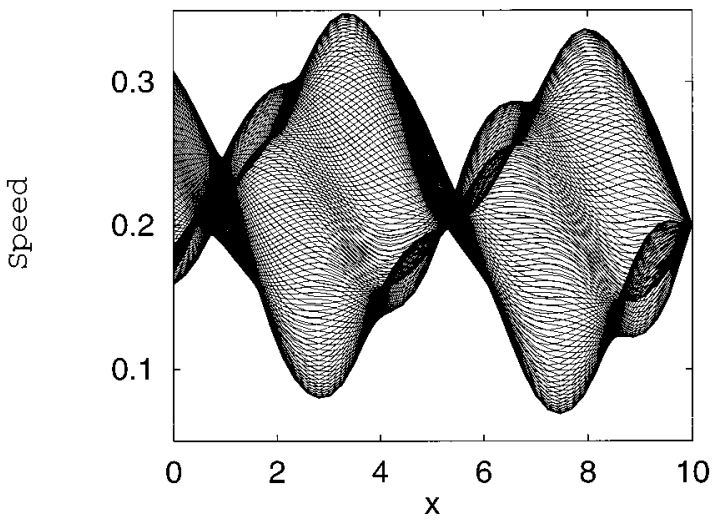

FIG. 7. Exponentially shaped junction: local speed $(\partial \phi / \partial t) /(\partial \phi / \partial x)$ as a function of $x$ for different times, same parameters as in Fig. 4.

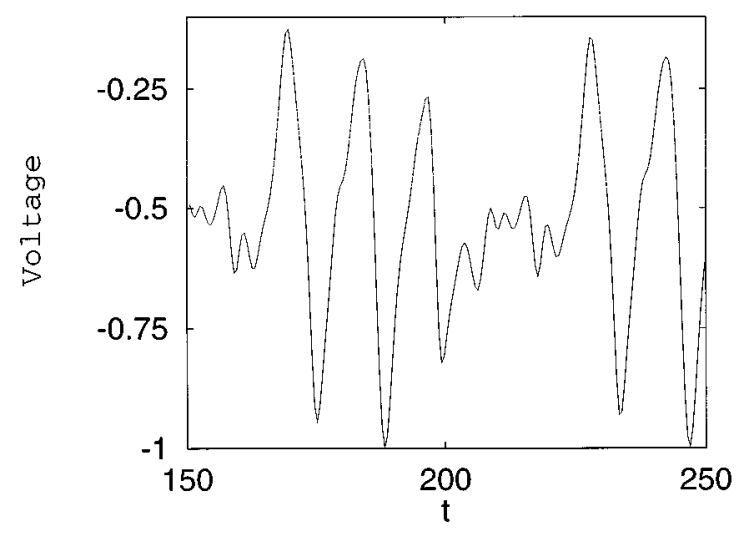

FIG. 8. Voltage in the middle of a rectangular junction vs time. Same parameters as for Fig. 4.

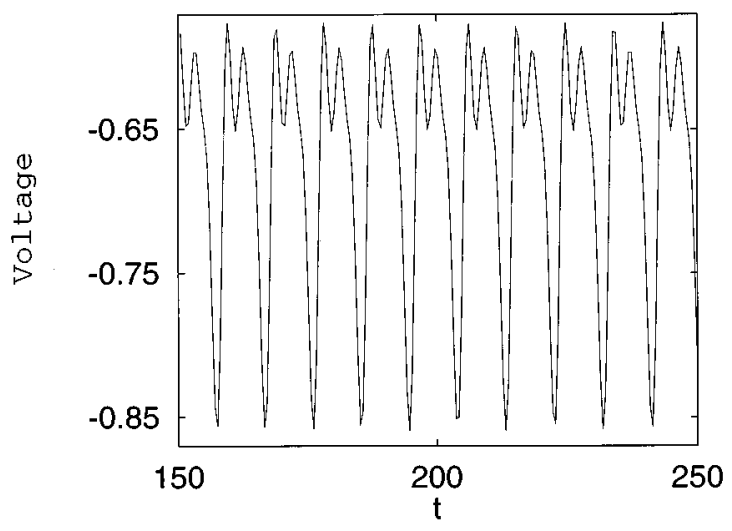

FIG. 9. Voltage in the middle of an exponentially shaped junction vs time. Same parameters as for Fig. 4.

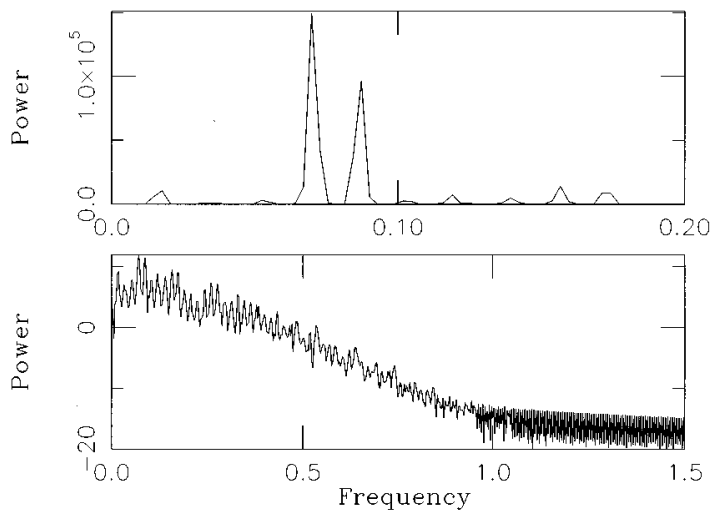

FIG. 10. Fourier power spectrum of voltage for the rectangular junction in linear-linear scales (top) and log-linear scales (bottom). The voltage is measured in the middle of the junction. The spectrum has been computed from 8192 data points. The parameters are the same as Fig. 4. 


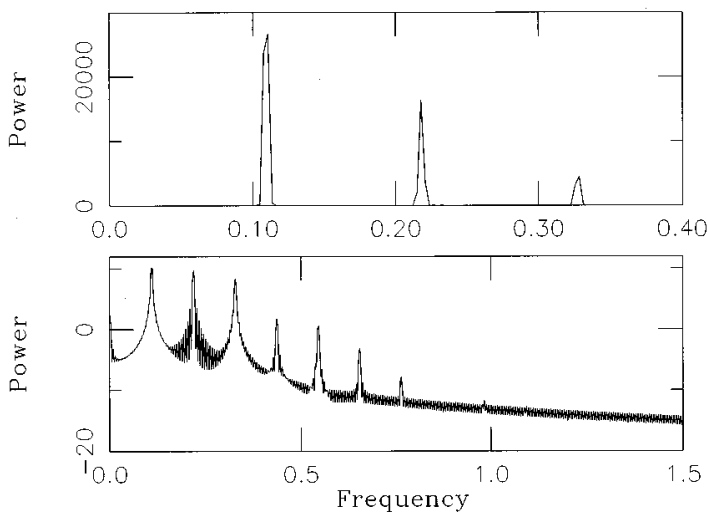

FIG. 11. Fourier power spectrum of voltage for the exponentially shaped junction in linear-linear scales (top) and log-linear scales (bottom). The voltage is measured in the middle of the junction. The spectrum has been computed from 8192 data points. The parameters are the same as Fig. 4.

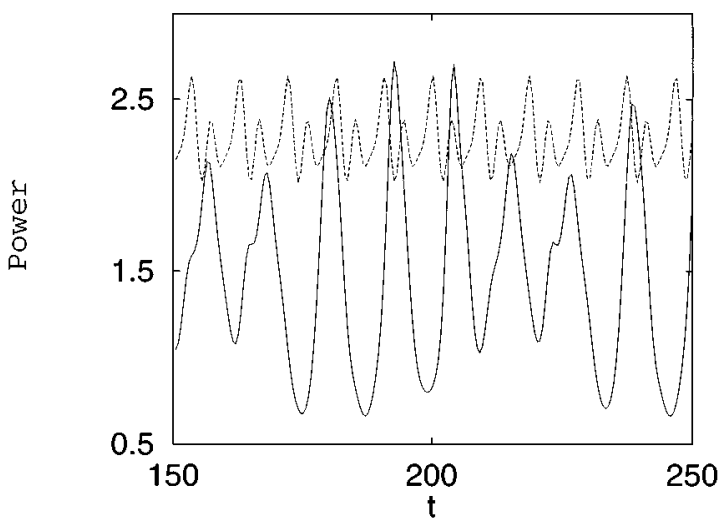

FIG. 12. Output power in the load $z(\partial \phi / \partial x)^{2}(l, t)$ vs time for a rectangular junction (full line) and an exponentially shaped junction (dashed line), the parameters are the same as in Figs. 4 and 6.

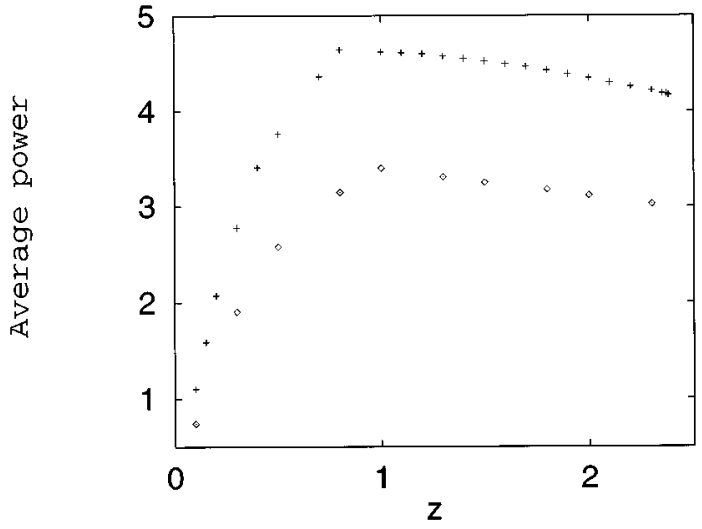

FIG. 13. Average output power vs the load $z$ for a rectangular junction $(\diamond)$ and an exponentially shaped junction $(+)$, the parameters are the same as in Figs. 4.

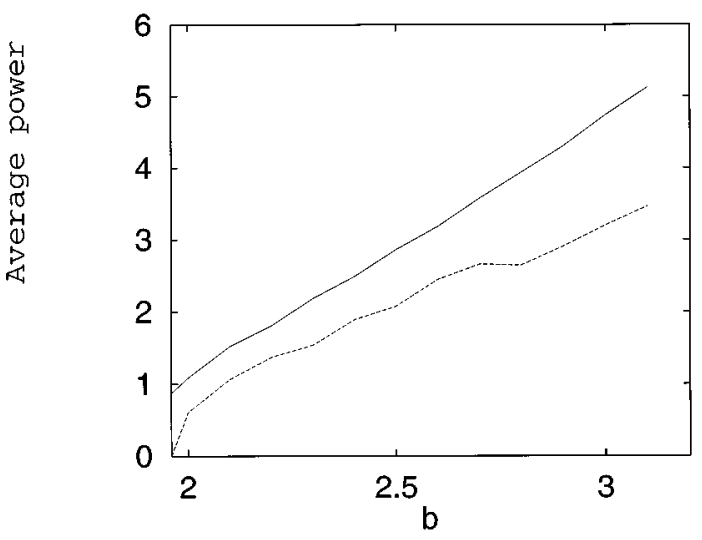

FIG. 14. Average output power vs the bias current $b$ for a rectangular junction in dashed line and an exponentially shaped junction in full line for the load $z=0.8$; the other parameters are the same as in Fig. 2.

load $z$ for the value of the bias current $b=3.1$, for the rectangular and exponentially shaped junctions studied in Figs. $7-12$. For both configurations the output power increases and becomes in a first approximation practically independent of the load, which is a desirable feature for using these devices as oscillators. Notice also that the average output power is clearly larger for the exponential profile and reaches its maximum value at about twice the matching impedance of $z=0.4$ which is a typical behavior for nonlinear oscillators. ${ }^{10}$ In Fig. 14 we plotted the average output power as a function of the bias current $b$ for the impedance realizing the maximum, $z=0.8$. Again we find the exponential design gives more output power, and the difference increases with the bias current.

\section{CONCLUSIONS}

We have shown that a highly regular, tunable oscillator can be made by applying a bias current to the larger end of an exponentially tapered long Josephson junction. Compared with a rectangular geometry, this design has smaller linewidth, increased output power, and better impedance matching to a load. The absence of external magnetic field -which can be an important source of spectral

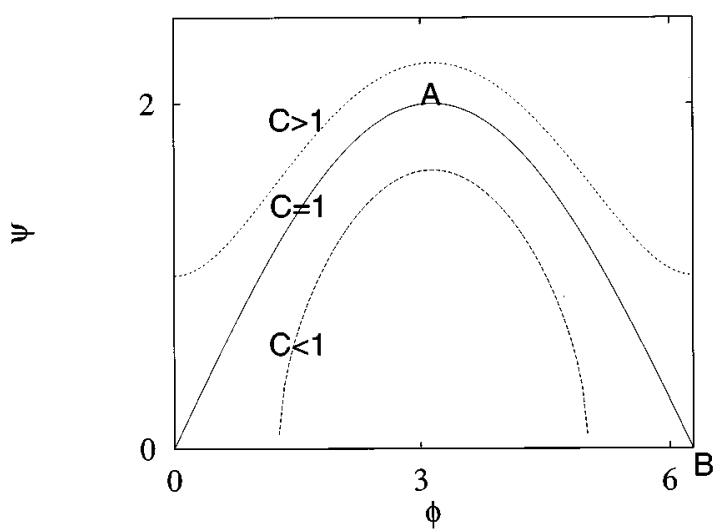

FIG. 15. First quadrant of the phase plane $(\phi, \widetilde{\psi})$ associated with the static problem Eq. (20) where $\lambda=0$. 
broadening - is also a big advantage.

Future directions for this study will consider the following questions: (i) How does output power depend on the load impedance? (ii) Can the addition of an external circuit to the device increase the power into the load? (iii) What is the influence at high frequency of surface damping (the $\beta$ term) $?^{3,7}$

\section{ACKNOWLEDGMENTS}

We gratefully acknowledge the support of European Network Grant No. CHRX-CT93-0331. A.B. and J.G.C. thank Mads Peter Soerensen for useful discussions.

\section{APPENDIX: THE STATIC CASE}

Equation (20) can be solved in the case $\lambda=0$ in terms of elliptic functions. ${ }^{11}$ First multiply the equation by $d \phi / d x$ and integrate to yield

$$
\frac{d \phi}{d x}= \pm \sqrt{2(C-\cos \phi)}
$$

where $C$ is a constant and the boundary conditions Eqs. (21), (22) require the positive sign. Introducing $k$ by $C=\left(2-k^{2}\right) / k^{2}$ we obtain

$$
\frac{d \phi}{d x}=\frac{2}{k} \sqrt{1-k^{2} \sin ^{2}\left(\frac{\phi}{2}-\frac{\pi}{2}\right)},
$$

which can be integrated from $\pi$ to $\Phi$ to yield

$$
\frac{k}{2} \int_{\pi}^{\Phi} \frac{d \phi}{\sqrt{1-k^{2} \sin ^{2}\left(\frac{\phi}{2}-\frac{\pi}{2}\right)}}=x(\Phi)-x_{\pi} .
$$

Introducing the change of variables $\psi=(\phi-\pi) / 2$

$$
\int_{0}^{(\Phi-\pi) / 2} \frac{d \psi}{\sqrt{1-k^{2} \sin ^{2} \psi}}=\frac{x(\Phi)-x_{\pi}}{k},
$$

which is the definition of the a.m. elliptic function, we get

$$
\begin{gathered}
\frac{\Phi}{2}-\frac{\pi}{2}=\text { a.m. }\left(\frac{x(\Phi)-x_{\pi}}{k} ; k^{2}\right), \\
\sin \left(\frac{\Phi}{2}-\frac{\pi}{2}\right)=\operatorname{sn}\left(\frac{x(\Phi)-x_{\pi}}{k} ; k^{2}\right), \\
\cos \left(\frac{\Phi}{2}-\frac{\pi}{2}\right)=\operatorname{cn}\left(\frac{x(\Phi)-x_{\pi}}{k} ; k^{2}\right),
\end{gathered}
$$

and

$$
\frac{k}{2} \frac{d \phi}{d x}=\sqrt{1-k^{2} \sin ^{2}\left(\frac{\Phi}{2}-\frac{\pi}{2}\right)}=d n\left(\frac{x(\Phi)-x_{\pi}}{k} ; k^{2}\right) .
$$

The boundary condition Eq. (22) at $x=l$ implies

$$
0=d n\left(\frac{l-x_{\pi}}{k} ; k^{2}\right)
$$

which can only be satisfied for $k=1 .{ }^{13}$ When $k=1$ elliptic functions reduce to hyperbolic functions so that Eqs. (A4)(A7) become

$$
\begin{gathered}
\frac{d \Phi}{d x}(x)=2 \operatorname{sech}\left(x-x_{\pi}\right), \\
\sin \left(\frac{\Phi}{2}\right)=\frac{1}{\cosh \left(x(\Phi)-x_{\pi}\right)},
\end{gathered}
$$

and

$$
\cos \left(\frac{\Phi}{2}\right)=-\tanh \left[x(\Phi)-x_{\pi}\right]
$$

The boundary condition Eq. (21) at $x=0$ can be satisfied if

$$
x_{\pi}=\ln \left(\frac{2}{b}-\sqrt{\frac{4}{b^{2}}-1}\right),
$$

so the static solution is

$$
\Phi(x)=-2 \arctan \left(\frac{1}{\sinh \left(x-x_{\pi}\right)}\right) .
$$

It is important to notice that as the junction length is decreased, the value of $b$ for which a solution exists is decreased. This corresponds to the fact that the magnetic field penetrates easier into a short junction.

To show the influence of $\lambda$, it is useful to draw the phase plane $(\phi, \widetilde{\psi})$ associated with Eq. (20). The orbits are completely defined by Eq. (A1) and Fig. 15 shows their different type depending on the value of the energy constant $C$. We have only represented the upper right-hand quadrant, the rest can be completed by symmetry. Notice also the fixed points $\phi=n \pi$. The static solution Eq. (A10) corresponds to the section $A-B$ on the $C=1$ curve for which $\widetilde{\psi}=d \phi / d x=2$ and $\phi=\pi$ for $x=0$ and $d \phi / d x=0$ and $\phi=2 \pi$ for $x=l$. The position of the fixed points is independent of $\lambda$ so that it is natural to look for a solution in the form of an expansion $\phi_{0}+\lambda \phi_{1}$ where $\phi_{0}$ satisfies Eq. (A1) and is given by Eq. (A10). Multiplying Eq. (20) by $d \phi / d x$ we obtain

$$
\frac{d}{d x}\left[\frac{1}{2}\left(\frac{d \phi}{d x}\right)^{2}+\cos \phi\right]=\lambda\left(\frac{d \phi}{d x}\right)^{2},
$$

which can be integrated from 0 to $l$

$$
\left[\frac{1}{2}\left(\frac{d \phi}{d x}\right)^{2}+\cos \phi\right]_{0}^{l}=\int_{0}^{l} \lambda\left(\frac{d \phi}{d x}\right)^{2} d x .
$$

We are looking for the maximum value of $b$ which corresponds to the $A-B$ orbit for $\phi_{0}$ so that $\phi_{0}(0)=\pi$ and $\phi_{0}(l)=0$. Using Eq. (A9) the right-hand side in the above equation can be evaluated at order $\lambda$ to yield

$$
\int_{0}^{l}\left(\frac{d \phi}{d x}\right)^{2} d x=4 \int_{0}^{l} \frac{d x}{\cosh ^{2} x}+O(\lambda)=4+O(\lambda) .
$$

Combining the above relations yields the behavior of the maximum value of $b$

$$
b_{m}=2(1-\lambda)+O\left(\lambda^{2}\right) .
$$


${ }^{1}$ Nonlinear Superconducting Devices and High $T_{c}$ Materials, edited by R. D. Parmentier and N. F. Pedersen (World Scientific, Singapore, 1995).

${ }^{2}$ A. V. Shchukin, V. P. Koshelets, and J. Mygind, in Nonlinear Superconducting Devices and High $T_{c}$ Materials (Ref. 1), p. 403.

${ }^{3}$ V. P. Koshelets, A. V. Shchukin, I. L. Lapytskaya, and J. Mygind, Phys. Rev. B 51, 6536 (1995).

${ }^{4}$ S. Pagano et al., in Nonlinear Superconducting Devices and High $T_{c}$ Materials (Ref. 1), p. 437.

${ }^{5}$ A. G. Lindgren and R. J. Buratti, Trans. IEEE Circuit Theory, 16, 274 (1969).

${ }^{6}$ D. W. Thompson, On Growth and Form, abridged edition (Cam- bridge University Press, Cambridge, 1966).

${ }^{7}$ A. C. Scott, Solid State Electron. 7, 137 (1964).

${ }^{8}$ A. Barone and G. Paterno, Physics and Applications of the Josephson Effect (Wiley, New York, 1982).

${ }^{9}$ D. W. McLaughlin and A. C. Scott, Phys. Rev. A 18, 1652 (1978).

${ }^{10}$ A. C. Scott, Active and Nonlinear Wave Propagation in Electronics (Wiley, New York, 1970).

${ }^{11}$ C. S. Owen and D. J. Scalapino, Phys. Rev. 164, 538 (1967).

${ }^{12}$ J. G. Caputo, N. Flytzanis, and M. Vavalis, Int. J. Mod. Phys. C 7, 191 (1996).

${ }^{13}$ B. F. Byrd and M. D. Friedman, Handbook of Elliptic Integrals for Engineers and Physicists (Springer, Berlin, 1954). 